This is the post print version of the article, which has been published in Journal of trace elements in medicine and biology . 2018, 48, 149-156. http://dx.doi.org/ 10.1016/j.jtemb.2018.03.014 .

\title{
GENE EXPRESSION IN ADVERSE REACTION TO METAL DEBRIS AROUND METAL-ON-METAL
}

ARTHROPLASTY: AN RNA-SEQ-BASED STUDY

Short title: Gene expression in adverse reaction to metal debris: an RNA-Seq study

This document has been downloaded from TamPub.uta.fi

The Institutional Repository of University of Tampere

Antti Pemmari ${ }^{1}$, Tiina Leppänen ${ }^{1}$, Erja-Leena Paukkeri ${ }^{1}$, Antti Eskelinen ${ }^{2}$, Teemu Moilanen ${ }^{1,2}$ and

\section{Eeva Moilanen ${ }^{1 *}$}

${ }^{1}$ The Immunopharmacology Research Group, Faculty of Medicine and Life Sciences, University of

Tampere and Tampere University Hospital, Tampere, Finland

${ }^{2}$ Coxa Hospital for Joint Replacement, Tampere, Finland

* Corresponding author

eeva.moilanen@staff.uta.fi (EM)

Mailing address:

University of Tampere

Faculty of Medicine and Life Sciences

Arvo-Building

Arvo Ylpön katu 34

33014 Tampere

Finland 


\section{Abstract}

Joint replacement surgery is a standard treatment of advanced osteoarthritis (OA). Since 2000, cobaltchromium (CoCr) metal-on-metal (MoM) implants were widely used in hip arthroplasties. Some patients developed "adverse reaction to metal debris" (ARMD) around the prosthesis resulting in a need for revision surgery. In the present study, we addressed the pathogenesis of ARMD by genome-wide expression analysis.

Pseudosynovial ARMD tissue was obtained from revision surgery of Articular Surface Replacement (ASR, DePuy, Warsaw, IN, USA) hip arthroplasties. Control tissue was 1) OA synovium from primary hip arthroplasties and 2) inflammatory pseudosynovial tissue from metal-on-plastic (MoP) implant revisions.

In ARMD tissue, the expression of 1446 genes was significantly increased and that of 1881 decreased as compared to OA synovium. Genes associated with immune response, tissue development and certain leukocyte signaling pathways were enriched in the differently $(F C>2)$ expressed genes. The network analysis proposed PRKACB, CD2, CD52 and CD53 as the central regulators of the greatest (FC >10) differences.

When ARMD tissue was compared to MoP tissue, the expression of 16 genes was significantly higher and that of 21 lower. Many of these genes were associated with redox homeostasis, metal ion binding and transport, macrophage activation and apoptosis. Interestingly, genes central to myofibroblast (AEBP1 and DES) and osteoclast (CCL21, TREM2 and CKB) development were upregulated in the MoP tissue. In network analysis, IL8, NQO1, GSTT1 and HMOX1 were identified as potential central regulators of the changes.

In conclusion, excessive amounts of CoCr debris produced by MoM hip implants induces in a group of patients a unique adverse reaction characterized with enhanced expression of genes associated with inflammation, redox homeostasis, metal ion binding and transport, macrophage activation and apoptosis.

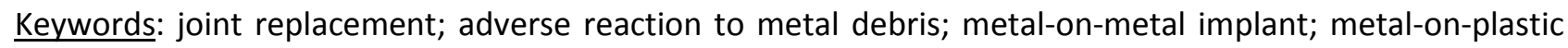
implant; RNA-Seq 


\section{Introduction}

Total hip joint replacement surgery is a standard treatment for advanced osteoarthritis (OA), rheumatoid arthritis (RA) and hip fracture [1-3]. During the first decade of the $21^{\text {st }}$ century, metal-on-metal (MoM) implants were widely used in these operations, aiming at increased mechanical durability compared to the conventional metal-on-plastic (MoP) implants $[4,5]$. However, a part of the patients developed adverse inflammatory reactions around the implant, requiring revision surgery. Named "adverse reaction to metal debris" (ARMD), these reactions are characterized by marked inflammation and, in some cases, pseudotumor formation $[6,7]$.

The ARMD reaction is thought to be caused by metal, especially cobalt, ions and nanoparticles abraded from the implant $[8,9]$, but the detailed pathogenesis of the reaction remains unknown. However, it has been shown to include systemic dissemination of metal ions and nanoparticles, increased oxidative stress, inflammation, DNA damage and coagulative necrosis [10-12].

Lymphocytes and macrophages are predominant cell types in ARMD reaction [6,13]. Metal ions may act as haptens, activating T cells and eliciting a delayed hypersensitivity reaction (type IV immune response) [14]. Their direct cytotoxic effects can also cause tissue necrosis, which in turn may attract macrophages and lead to granulomatous responses [15] and osteolysis [16]. Cobalt may also stimulate macrophages through direct activation of Toll-like receptor 4 (TLR4) [17] and/or so-called danger signaling [18].

Reactions observed around a failed MoP implants share many features of the ARMD reaction. They are characterized by lymphocytic inflammation [19], macrophage activation, differentiation of mononuclear cells into osteoclasts and subsequent osteolysis [20]. These reactions are thought to be driven by implant-derived polyethylene particles [21], which can elicit inflammation and osteolysis in in vitro models [22].

In the present study, we approached the pathogenesis of the ARMD reaction by genome-wide expression analysis, with a special focus on differences between ARMD tissue and the inflammatory response around failed MoP joints. 


\section{Materials and methods}

\section{Patients}

The study was approved by the Ethics Committee of Tampere University Hospital, Tampere, Finland, and complies with the declaration of Helsinki. All patients provided their written informed consent. Pseudotumor tissue from ten revision surgeries of Articular Surface Replacement (ASR) XL hip implants (DePuy, Warsaw, IN, USA) were collected and analyzed. Control samples of pseudosynovial tissue were collected from six revision operations of failed metal-on-plastic (MoP) joints and synovial samples from five OA patients in primary total hip arthroplasties. All operations were carried out at Coxa Hospital for Joint Replacement, Tampere, Finland, and all primary arthroplasties had been performed for the treatment of end-stage osteoarthritis.

\section{Reasons for Revision Surgery}

Revision surgeries of MoM hips were performed for one (or more) of the following indications: 1) a pseudotumour, either with a solid core or atypical contents, was seen in the vicinity of the implant, regardless of symptoms and whole blood metal ion levels; or 2) the patient had both elevated metal ion levels and hip symptoms despite a normal finding on cross-sectional imaging; or 3) the patient had an increasingly and significantly symptomatic hip regardless of imaging findings or metal ion levels. Symptoms included hip pain, discomfort, sense of instability, and/or impaired function of the hip as well as sounds from the hip. Infection was ruled out by at least five bacterial cultures obtained during revision surgery. The revision surgeries of MoP hips were performed for either aseptic loosening of the implants or for recurrent dislocation of the hip. The MoP implants included various brands and had been in-situ for a minimum of one year.

\section{Tissue processing and RNA extraction}

Peri-implant tissue was obtained directly from surgery. Necrotic mass (if present) was removed, and the tissue was cut into pieces weighing approximately $100 \mathrm{mg}$ and the tissue samples were stored in $1000 \mu \mathrm{l}$ of 
RNAlater $^{\mathrm{TM}}$ solution (Thermo Fisher Scientific, Waltham, MA, USA). The samples were centrifuged, supernatant removed, and the samples were homogenized in QIAshredder ${ }^{\mathrm{TM}}$ columns (Qiagen). Total RNA was extracted using RNeasy Mini Spin columns (Qiagen) and treated with DNAse (Fermentas UAB, Vilnius, Lithuania).

Next-generation sequencing and data analysis

Sequencing of the RNA samples was performed in the Turku Centre of Biotechnology sequencing core, Turku, Finland, using the Illumina HiSeq 2500 sequencing platform. Sequencing depth was 20 million single-end reads with length of 50 base pairs (bp). The data was analyzed using the automated TRAPLINE RNA-Seq data analysis workflow [23] implemented on the Galaxy platform [24]. In brief, the reads were trimmed for quality, and read quality was assessed using FastQC [25]. The reads were aligned to a reference human genome using TopHat2 [26], and differential expression was assessed with Cufflinks [27]. For the purposes of further analysis, genes with an expression fold change $(F C)>2.0$ in either direction and false discovery rate (FDR)corrected $p$-value $<0.05$ were deemed biologically and statistically significant. Functions of the genes were obtained from the NCBI Gene database, if not otherwise indicated. Mean gene expression levels are reported as reads per kilobase per million (RPKM) values. Functional analysis from the Gene Ontology (GO) database [28] was performed using the DAVID tool [29], and the resulting list was reduced using REVIGO [30]. Protein interactions were studied with STRING [31]. 


\section{Results}

When comparing ARMD tissue to OA tissue, the expression of 1446 genes was found to be significantly higher and that of 1881 genes significantly lower in the former. Of these, 622 genes had a positive expression fold change (FC) of more than 2.0, and 528 a negative one of similar magnitude. Tables 1 and 2 show 20 genes with the greatest FCs into both directions, along with their functions potentially relevant for the metal debrisinduced reaction. These can be seen to encompass a wide variety of different actions, especially lymphocyte and macrophage-mediated inflammatory response, tissue development, redox homeostasis and cellular metabolism. 
Table 1: 20 genes with the highest expression levels in ARMD compared to OA tissue. Gene expression levels in adverse reaction to metal debris (ARMD) and osteoarthritis (OA) samples are listed as reads per kilobase million (RPKM) values, and the differences as fold changes (FCs). $p$-values are adjusted by false discovery rate (FDR).

\begin{tabular}{|c|c|c|c|c|c|c|}
\hline Gene & Name & Function & RPKM (ARMD) & RPKM (OA) & FC & adj. $p$ \\
\hline TNFRSF14 & $\begin{array}{l}\text { TNF receptor superfamily } \\
\text { member } 14\end{array}$ & T-cell mediated immunity & 61.09 & 0.20 & 307.19 & 0.0018 \\
\hline FAM213B & $\begin{array}{l}\text { Family with sequence } \\
\text { similarity } 213 \text { member B }\end{array}$ & Prostaglandin synthesis & 271.81 & 1.14 & 239.05 & 0.00043 \\
\hline KCNAB2 & $\begin{array}{l}\text { Potassium voltage-gated } \\
\text { channel subfamily A } \\
\text { regulatory beta subunit } 2\end{array}$ & Potassium transport & 18.57 & 0.10 & 184.80 & 0.042 \\
\hline PGD & \begin{tabular}{|l|}
$\begin{array}{l}\text { Phosphogluconate } \\
\text { dehydrogenase }\end{array}$ \\
\end{tabular} & Pentose phosphate shunt & 33.22 & 0.22 & 153.04 & 0.014 \\
\hline PEX14 & \begin{tabular}{|l|} 
Peroxisomal biogenesis \\
factor 14
\end{tabular} & Peroxisome production & 3452.48 & 42.31 & 81.59 & 0.00043 \\
\hline AGTRAP & $\begin{array}{l}\text { Angiotensin II receptor } \\
\text { associated protein }\end{array}$ & $\begin{array}{l}\text { Regulation of vascular } \\
\text { tone }\end{array}$ & 187.96 & 2.44 & 77.09 & 0.00043 \\
\hline MIIP & $\begin{array}{l}\text { Migration and invasion } \\
\text { inhibitory protein } \\
\end{array}$ & \begin{tabular}{|l|}
$\begin{array}{l}\text { Regulation of cell } \\
\text { migration }\end{array}$ \\
\end{tabular} & 16.12 & 0.24 & 66.56 & 0.00043 \\
\hline TMEM51 & Transmembrane protein 51 & Membrane component & 54.35 & 0.96 & 56.90 & 0.00043 \\
\hline EFHD2 & $\begin{array}{l}\text { EF-hand domain family } \\
\text { member D2 }\end{array}$ & \begin{tabular}{|l|} 
Regulation of cell \\
migration
\end{tabular} & 48.94 & 0.88 & 55.36 & 0.00043 \\
\hline PLEKHM2 & \begin{tabular}{|l|} 
Pleckstrin homology and \\
RUN domain containing M2
\end{tabular} & Organelle localization & 128.21 & 2.63 & 48.69 & 0.00043 \\
\hline NECAP2 & $\begin{array}{l}\text { NECAP endocytosis } \\
\text { associated } 2 \\
\end{array}$ & Endocytosis & 45.97 & 0.99 & 46.31 & 0.00043 \\
\hline ARHGEF10L & \begin{tabular}{|l|}
$\begin{array}{l}\text { Rho guanine nucleotide } \\
\text { exchange factor } 10 \text { like }\end{array}$ \\
\end{tabular} & Signal transduction & 55.92 & 1.37 & 40.81 & 0.00043 \\
\hline C1QA & Complement C1q A chain & Innate immunity & 16.80 & 0.43 & 38.84 & 0.00043 \\
\hline PITHD1 & PITH domain containing 1 & $\begin{array}{l}\begin{array}{l}\text { Regulation of gene } \\
\text { expression }\end{array} \\
\end{array}$ & 1916.92 & 54.85 & 34.95 & 0.00043 \\
\hline LYPLA2 & Lysophospholipase II & Lipid metabolism & 20.83 & 0.60 & 34.93 & 0.0024 \\
\hline SH3BGRL3 & $\begin{array}{l}\text { SH3 domain binding } \\
\text { glutamate rich protein like } 3\end{array}$ & Redox homeostasis & 43.63 & 1.30 & 33.68 & 0.00043 \\
\hline CD52 & CD52 molecule & $\begin{array}{l}\text { Respiratory burst, T cell } \\
\text { receptor signaling }\end{array}$ & 19.91 & 0.60 & 32.93 & 0.00043 \\
\hline ZDHHC18 & $\begin{array}{l}\text { Zinc finger DHHC-type } \\
\text { containing } 18\end{array}$ & $\begin{array}{l}\text { Posttranslational } \\
\text { modification }\end{array}$ & 15.60 & 0.49 & 32.09 & 0.00043 \\
\hline SYTL1 & Synaptotagmin like 1 & Exocytosis & 4043.20 & 137.35 & 29.44 & 0.00043 \\
\hline RAB42 & $\begin{array}{l}\text { RAB42, member RAS } \\
\text { oncogene family }\end{array}$ & $\begin{array}{l}\text { GTPase activity, GTP } \\
\text { binding }\end{array}$ & 11.91 & 0.44 & 27.17 & 0.0069 \\
\hline
\end{tabular}


Table 2: 20 genes with the lowest expression levels in ARMD compared to OA tissue. Gene expression levels in adverse reaction to metal debris (ARMD) and osteoarhtritis (OA) samples are listed as reads per kilobase million (RPKM) values, and the differences as fold changes (FCs). $p$-values are adjusted by false discovery rate (FDR).

\begin{tabular}{|c|c|c|c|c|c|c|}
\hline Gene & Name & Function & \begin{tabular}{|l|} 
RPKM \\
(ARMD)
\end{tabular} & $\begin{array}{l}\text { RPKM } \\
\text { (OA) }\end{array}$ & FC & adj. $p$ \\
\hline UCK2 & Uridine-cytidine kinase 2 & Pyrimidine metabolism & 4.66 & 1387.25 & -297.99 & 0.00043 \\
\hline GDF5 & Growth differentiation factor 5 & Bone and cartilage development & 0.15 & 19.56 & -131.23 & 0.014 \\
\hline GPD1 & $\begin{array}{l}\text { Glycerol-3-phosphate } \\
\text { dehydrogenase } 1\end{array}$ & $\begin{array}{l}\text { Carbohydrate and lipid } \\
\text { metabolism }\end{array}$ & 0.18 & 22.04 & -122.14 & 0.00043 \\
\hline ADH1B & $\begin{array}{l}\text { Alcohol dehydrogenase 1B } \\
\text { (class I), beta polypeptide }\end{array}$ & Alcohol metabolism & 0.22 & 21.45 & -98.64 & 0.00043 \\
\hline SCUBE1 & $\begin{array}{l}\text { Signal peptide, CUB domain } \\
\text { and EGF like domain containing } \\
1\end{array}$ & Thrombosis and inflammation & 0.22 & 14.25 & -63.50 & 0.0008 \\
\hline TMEM196 & Transmembrane protein 196 & Regulation of cell proliferation & 0.41 & 17.98 & -43.38 & 0.00043 \\
\hline SCRG1 & Stimulator of chondrogenesis 1 & Chondrogenesis & 6.64 & 268.27 & -40.39 & 0.00043 \\
\hline NTRK2 & $\begin{array}{l}\text { Neurotrophic receptor tyrosine } \\
\text { kinase } 2\end{array}$ & Neuron development & 0.63 & 20.82 & -32.81 & 0.00043 \\
\hline AMTN & Amelotin & Cell adhesion & 3.89 & 123.23 & -31.67 & 0.00043 \\
\hline SEMA3A & Semaphorin $3 \mathrm{~A}$ & Inhibition of angiogenesis & 0.48 & 13.97 & -28.96 & 0.00043 \\
\hline ZNF385B & Zinc finger protein 385B & Apoptosis & 1.01 & 28.69 & -28.48 & 0.00043 \\
\hline FGF10 & Fibroblast growth factor 10 & Skeletal system development & 1.94 & 53.45 & -27.57 & 0.00043 \\
\hline DLX4 & Distal-less homeobox 4 & Regulation of transcription & 0.94 & 25.10 & -26.79 & 0.00739 \\
\hline GPR1 & G protein-coupled receptor 1 & $\begin{array}{l}\text { G protein coupled receptor } \\
\text { activity }\end{array}$ & 2.34 & 58.64 & -25.08 & 0.00043 \\
\hline CA9 & Carbonic anhydrase 9 & Cell proliferation & 2.30 & 56.10 & -24.35 & 0.00043 \\
\hline SLPI & $\begin{array}{l}\text { Secretory leukocyte peptidase } \\
\text { inhibitor }\end{array}$ & Immune response & 9.51 & 222.85 & -23.43 & 0.00043 \\
\hline CLIC5 & Chloride intracellular channel 5 & Chloride transport & 1.58 & 35.39 & -22.34 & 0.00043 \\
\hline SMOC1 & $\begin{array}{l}\text { SPARC related modular calcium } \\
\text { binding } 1\end{array}$ & Skeletal system development & 3.06 & 62.38 & -20.38 & 0.00043 \\
\hline STAC2 & SH3 and cysteine rich domain 2 & Metal ion binding & 0.54 & 10.61 & -19.76 & 0.00043 \\
\hline SGCA & Sarcoglycan alpha & Muscle development & 1.23 & 23.14 & -18.82 & 0.0008 \\
\hline DLX3 & Distal-less homeobox 3 & Blood vessel development & 1.20 & 21.90 & -18.31 & 0.00043 \\
\hline RASD1 & $\begin{array}{l}\text { Ras related dexamethasone } \\
\text { induced } 1\end{array}$ & Regulation of cell proliferation & 4.38 & 79.12 & -18.08 & 0.00043 \\
\hline
\end{tabular}


When functions of the genes with FC $>2.0$ were studied using the GO database (Table 3 ), functional categories involved immune response, macrophage and lymphocyte activation, cell adhesion, skeletal system development and several leukocyte signaling pathways (such as PI3KR1, phospholipase C, tyrosine kinase and integrin signaling). Additionally, Table 4 shows significant inflammatory and hypoxia-related genes which were expressed at higher level in ARMD than OA tissue.

When interactions between the most strongly up- and downregulated (FC > 10) genes were investigated, PRKACB, CD2, CD52 and CD53 were identified as potential central regulators of the observed changes in gene expression. The interaction network also contained the immunoglobulin receptor genes FCGR2A, FCGF2B and FCER1G. Another, smaller network was centered on the pentose phosphate shunt -related gene phosphogluconate dehydrogenase (PGD), and yet another on aggrecan (ACAN) (Figure 1). 
Table 3: GO terms covering genes with significantly altered expression in ARMD vs OA tissue. Genes with an expression fold change $(\mathrm{FC})>2.0$ in either direction were studied with the DAVID tool using the Gene Ontology (GO) database, and the resulting list of terms was reduced with REVIGO. $p$-values are corrected by false discovery rate (FDR).

\begin{tabular}{|c|c|c|c|}
\hline Term & $\begin{array}{l}\text { Number of } \\
\text { altered } \\
\text { genes }\end{array}$ & $\begin{array}{l}\text { Total number } \\
\text { of genes in } \\
\text { the term } \\
\end{array}$ & adj. $p$ \\
\hline Inflammatory response & 110 & 407 & $6.74 \mathrm{E}-21$ \\
\hline Cell adhesion & 117 & 751 & $1.21 \mathrm{E}-17$ \\
\hline Signal transduction & 199 & 4491 & $2.30 \mathrm{E}-10$ \\
\hline Adaptive immune response & 43 & 194 & $4.68 \mathrm{E}-07$ \\
\hline Cell-cell signaling & 59 & 338 & 5.94E-06 \\
\hline Cell surface receptor signaling pathway & 62 & 1997 & 8.90E-06 \\
\hline T cell costimulation & 28 & 73 & 1.09E-05 \\
\hline Integrin-mediated signaling pathway & 32 & 77 & $1.33 \mathrm{E}-05$ \\
\hline Positive regulation of protein kinase B signaling & 29 & 89 & 1.50E-05 \\
\hline Negative chemotaxis & 17 & 34 & 9.47E-05 \\
\hline $\begin{array}{l}\text { Transmembrane receptor protein tyrosine kinase signaling } \\
\text { pathway }\end{array}$ & 30 & 481 & $9.88 \mathrm{E}-05$ \\
\hline Extracellular matrix organization & 47 & 292 & 0.0001 \\
\hline Response to lipopolysaccharide & 35 & 260 & 0.00025 \\
\hline Positive regulation of GTPase activity & 98 & 629 & 0.00035 \\
\hline $\begin{array}{l}\text { Negative regulation of axon extension involved in axon } \\
\text { guidance }\end{array}$ & 14 & 230 & 0.00071 \\
\hline Aging & 46 & 291 & 0.00097 \\
\hline Positive regulation of interleukin-1 beta secretion & 13 & 26 & 0.0011 \\
\hline Positive chemotaxis & 16 & 33 & 0.0011 \\
\hline Positive regulation of cytosolic calcium ion concentration & 35 & 235 & 0.0024 \\
\hline Cellular defense response & 21 & 56 & 0.0027 \\
\hline Semaphorin-plexin signaling pathway & 15 & 22 & 0.0031 \\
\hline Positive regulation of cell proliferation & 81 & 832 & 0.015 \\
\hline Angiogenesis & 46 & 245 & 0.016 \\
\hline Positive regulation of peptidyl-tyrosine phosphorylation & 24 & 166 & 0.023 \\
\hline Platelet activation & 29 & 108 & 0.023 \\
\hline Leukocyte migration & 30 & 284 & 0.026 \\
\hline Proton transport & 17 & 130 & 0.032 \\
\hline Cellular response to interleukin-1 & 23 & 67 & 0.033 \\
\hline Positive regulation of phosphatidylinositol 3-kinase signaling & 20 & 64 & 0.033 \\
\hline Skeletal system development & 32 & 155 & 0.038 \\
\hline Activation of phospholipase C activity & 12 & 25 & 0.043 \\
\hline Protein localization to cell surface & 11 & 23 & 0.0478 \\
\hline
\end{tabular}


Table 4: Inflammatory and hypoxia-related genes with higher expression in ARMD compared to OA tissue.

Gene expression levels are listed as reads per kilobase million (RPKM) values, and the differences as fold changes (FCs). $p$-values are adjusted by false discovery rate (FDR).

\begin{tabular}{|c|c|c|c|c|c|c|}
\hline Gene & Name & Function & $\begin{array}{l}\text { RPKM } \\
\text { (ARMD) }\end{array}$ & $\begin{array}{l}\text { RPKM } \\
\text { (OA) }\end{array}$ & FC & adj. $p$ \\
\hline \multicolumn{7}{|c|}{ Inflammatory genes } \\
\hline S100A9 & S100 calcium binding protein A9 & Innate immunity & 662.78 & 42.13 & 15.73 & 0.00043 \\
\hline FCGR2B & Fc fragment of IgG receptor IIb & Adaptive immune response & 17.80 & 1.30 & 13.67 & 0.00043 \\
\hline IL2RA & Interleukin 2 receptor subunit alpha & Lymphocyte activation & 10.14 & 1.55 & 6.54 & 0.00043 \\
\hline IL18 & Interleukin 18 & Immune response & 118.70 & 27.13 & 4.37 & 0.00043 \\
\hline TNFSF13B & TNF superfamily member $13 \mathrm{~b}$ & $\begin{array}{l}\text { Lymphocyte proliferation and } \\
\text { activation }\end{array}$ & 17.14 & 4.74 & 3.62 & 0.00043 \\
\hline SPN & Sialophorin & T cell activation & 38.79 & 12.96 & 2.99 & 0.00043 \\
\hline IRF8 & interferon regulatory factor 8 & Interferon-mediated immune response & 16.00 & 5.49 & 2.91 & 0.00043 \\
\hline CCL2 & $\mathrm{C}-\mathrm{C}$ motif chemokine ligand 2 & Inflammation, monocyte chemotaxis & 16.61 & 6.07 & 2.74 & 0.00043 \\
\hline CCL13 & C-C motif chemokine ligand 13 & Lymphocyte and monocyte chemotaxis & 27.02 & 9.93 & 2.72 & 0.00043 \\
\hline CCL18 & $\mathrm{C}-\mathrm{C}$ motif chemokine ligand 18 & Lymphocyte chemotaxis & 137.69 & 50.68 & 2.72 & 0.00043 \\
\hline CCR7 & $\mathrm{C}-\mathrm{C}$ motif chemokine receptor 7 & Leukocyte activation and chemotaxis & 18.95 & 7.31 & 2.59 & 0.00043 \\
\hline CD7 & CD7 molecule & Lymphocyte activation & 11.12 & 4.45 & 2.50 & 0.00043 \\
\hline IL27RA & Interleukin 27 receptor subunit alpha & T cell activation & 9.18 & 3.86 & 2.38 & 0.00043 \\
\hline FCAR & Fc fragment of IgA receptor & IgA-mediated immunity & 10.28 & 4.50 & 2.29 & 0.00043 \\
\hline TNFSF14 & TNF superfamily member 14 & T cell activation & 68.91 & 30.37 & 2.27 & 0.00043 \\
\hline ZAP70 & $\begin{array}{l}\text { Zeta chain of T cell receptor } \\
\text { associated protein kinase } 70\end{array}$ & Lymphocyte activation & 133.38 & 62.73 & 2.13 & 0.00043 \\
\hline CD8A & CD8a molecule & Tc cell activation & 31.41 & 15.10 & 2.08 & 0.0083 \\
\hline CD8B & CD8b molecule & Tc cell activation & 22.48 & 10.82 & 2.08 & 0.00043 \\
\hline IL1B & Interleukin 1 beta & Systemic inflammation & 60.34 & 29.12 & 2.07 & 0.00043 \\
\hline CD40 & CD40 molecule & Lymphocyte activation & 16.37 & 8.06 & 2.03 & 0.00043 \\
\hline \multicolumn{7}{|c|}{ Hypoxia-related genes } \\
\hline HMOX1 & Heme oxygenase 1 & Widespread response to hypoxia & 523.17 & 72.32 & 7.17 & 0.00043 \\
\hline GSTO1 & Glutathione S-transferase omega 1 & Prevention of oxidative injury & 411.06 & 60.58 & 6.79 & 0.00043 \\
\hline ASCL2 & $\begin{array}{l}\text { Achaete-scute family bHLH } \\
\text { transcription factor } 2\end{array}$ & Response to hypoxia, HIF1A pathway & 327.59 & 68.85 & 4.76 & 0.00043 \\
\hline TXNRD1 & Thioredoxin reductase 1 & $\begin{array}{l}\text { Prevention of oxidative injury, Nrf2 } \\
\text { pathway }\end{array}$ & 84.50 & 20.76 & 4.07 & 0.00043 \\
\hline PRKCB & Protein kinase $\mathrm{C}$ beta & Response to hypoxia & 26.71 & 8.82 & 3.03 & 0.00043 \\
\hline EGLN3 & $\begin{array}{l}\text { Egl-9 family hypoxia inducible factor } \\
3\end{array}$ & $\begin{array}{l}\text { Apoptosis, regulation of cell } \\
\text { proliferation }\end{array}$ & 31.72 & 11.33 & 2.81 & 0.00043 \\
\hline NQO1 & $\mathrm{NAD}(\mathrm{P}) \mathrm{H}$ quinone dehydrogenase 1 & Redox homeostasis, NO biosynthesis & 31.52 & 11.99 & 2.63 & 0.00043 \\
\hline SOD2 & Superoxide dismutase 2 & Superoxide detoxification & 356.58 & 135.92 & 2.62 & 0.00043 \\
\hline GPX4 & Glutathione peroxidase 4 & Prevention of oxidative injury & 10.67 & 4.35 & 2.45 & 0.00043 \\
\hline CXCR4 & $\mathrm{C}-\mathrm{X}-\mathrm{C}$ motif chemokine receptor 4 & Response to hypoxia, HIF1A pathway & 8.27 & 4.00 & 2.07 & 0.00043 \\
\hline
\end{tabular}




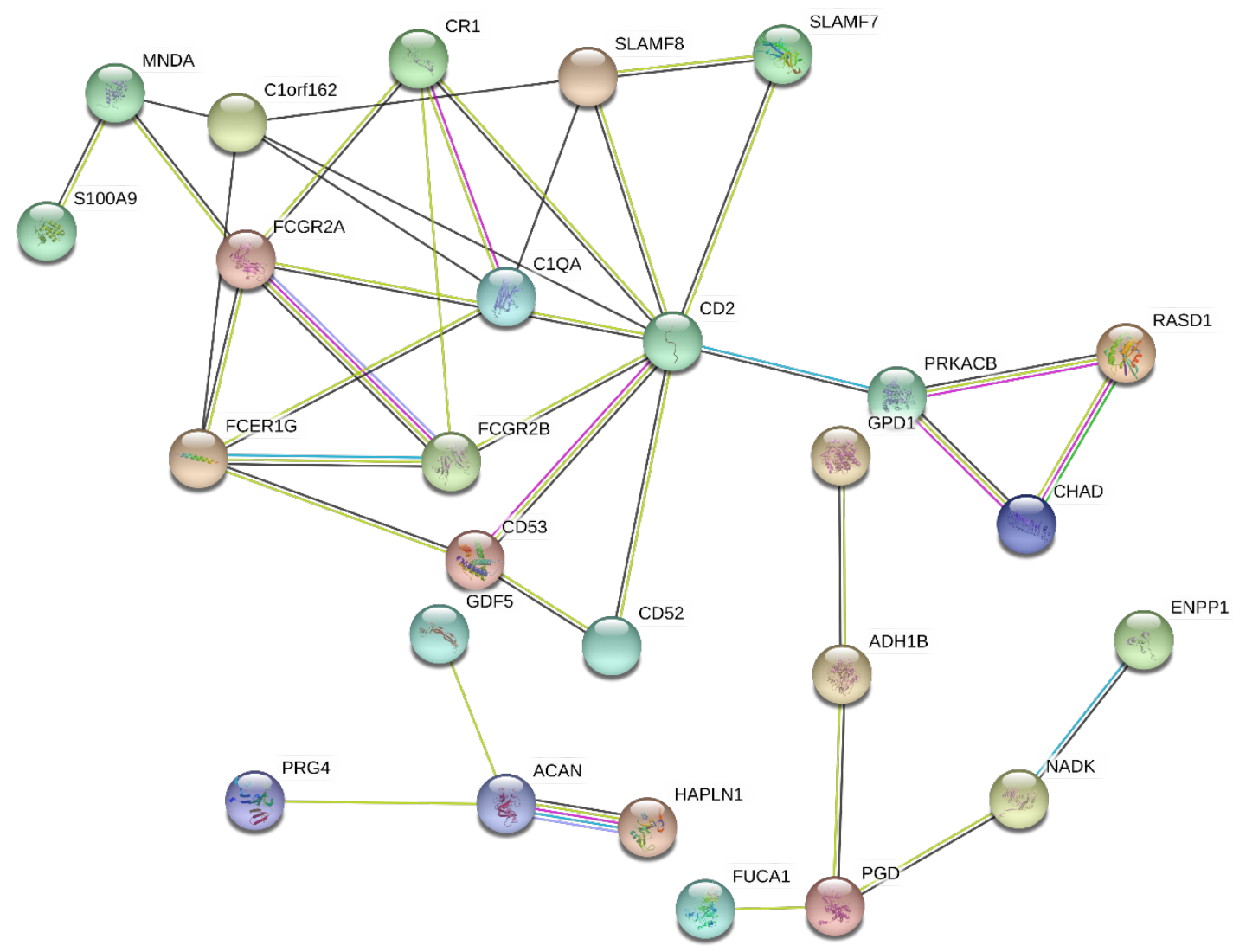

Figure 1: Interactions among the genes with greatest expression fold change in ARMD vs OA tissue. Genes with expression fold change $(F C)>10$ in adverse reaction to metal debris (ARMD) vs osteoarthritis $(O A)$ tissue were studied with STRING. Genes with no more than 2 interactions are excluded from the graph. Colors of the edges: green $=$ activation, blue $=$ binding, black $=$ chemical reaction, red $=$ inhibition, violet $=$ catalysis, pink $=$ posttranslational modification, yellow $=$ transcriptional regulation, grey $=$ other interaction . 
Next, we compared tissue from ARMD reaction to the inflammatory pseudosynovial tissue from a failed MoP joint. In these cases, the differences in gene expression were less pronounced: the expression of 16 genes was significantly higher (Table 5) and 21 significantly lower (Table 6) in the ARMD reaction. All of these genes had a FC of more than 2.0 into either direction. Interestingly, the expression of genes central to myofibroblast (AEBP1 and DES) and osteoclast (CCL21, TREM2 and CKB) development was higher in MoP tissue.

When studying the interactions of the genes which were differentially expressed between ARMD and MoP tissues (Figure 2), IL8, NQO1, GSTT1 and HMOX1 were found to occupy central positions in the network, suggesting them as potential central regulators of the observed changes. 
Table 5: Genes with significantly higher expression in ARMD than in MoP tissue. Gene expression levels in adverse reaction to metal debris (ARMD) and metal-on-plastic (MoP) samples are listed as reads per kilobase million (RPKM) values, and the differences as fold changes (FCs). $p$-values are adjusted by false discovery rate (FDR).

\begin{tabular}{|c|c|c|c|c|c|c|}
\hline Gene & Name & Function & $\begin{array}{l}\text { RPKM } \\
\text { (ARMD) } \\
\end{array}$ & \begin{tabular}{|l|} 
RPKM \\
(MoP) \\
\end{tabular} & FC & adj. $p$ \\
\hline RNF170 & Ring finger protein 170 & $\begin{array}{l}\text { Protein } \\
\text { ubiquitionation, } \\
\text { metal ion binding }\end{array}$ & 165.93 & 3.21 & 51.77 & 0.0085 \\
\hline TRIM25 & $\begin{array}{l}\text { Tripartite motif } \\
\text { containing } 25\end{array}$ & Immune response & 105.81 & 5.25 & 20.16 & 0.0085 \\
\hline FAM89A & $\begin{array}{l}\text { Family with sequence } \\
\text { similarity } 89 \text { member } A\end{array}$ & ? & 167.50 & 10.55 & 15.88 & 0.0085 \\
\hline SCRIB & $\begin{array}{l}\text { Scribbled planar cell } \\
\text { polarity protein }\end{array}$ & $\begin{array}{l}\text { Regulation of cell } \\
\text { proliferation, } \\
\text { apoptosis }\end{array}$ & 99.17 & 8.92 & 11.12 & 0.0085 \\
\hline CPSF1 & $\begin{array}{l}\text { Cleavage and } \\
\text { polyadenylation specific } \\
\text { factor } 1\end{array}$ & mRNA processing & 125.18 & 11.94 & 10.49 & 0.0085 \\
\hline STMN1 & Stathmin 1 & Signal transduction & 128.25 & 19.43 & 6.60 & 0.0085 \\
\hline CCL8 & $\begin{array}{l}\text { C-C motif chemokine } \\
\text { ligand } 8\end{array}$ & Immune response & 25.81 & 5.36 & 4.81 & 0.023 \\
\hline NOP56 & NOP56 ribonucleoprotein & rRNA processing & 135.68 & 32.39 & 4.19 & 0.0085 \\
\hline SLC40A1 & $\begin{array}{l}\text { Solute carrier family } 40 \\
\text { member } 1\end{array}$ & Metal ion transport & 168.55 & 40.79 & 4.13 & 0.0085 \\
\hline NQO1 & $\begin{array}{l}\text { NAD(P)H quinone } \\
\text { dehydrogenase } 1\end{array}$ & $\begin{array}{l}\text { Redox homeostasis, } \\
\text { NO biosynthesis }\end{array}$ & 31.52 & 9.24 & 3.41 & 0.0085 \\
\hline FGFBP2 & $\begin{array}{l}\text { Fibroblast growth factor } \\
\text { binding protein } 2\end{array}$ & $\begin{array}{l}\text { Tc cell -mediated } \\
\text { immunity? }\end{array}$ & 62.22 & 20.04 & 3.10 & 0.019 \\
\hline HMOX1 & Heme oxygenase 1 & $\begin{array}{l}\text { Redox and metal ion } \\
\text { homeostasis }\end{array}$ & 523.17 & 187.11 & 2.80 & 0.014 \\
\hline CLEC5A & $\begin{array}{l}\text { C-type lectin domain } \\
\text { containing } 5 \mathrm{~A}\end{array}$ & Immune response & 29.03 & 10.55 & 2.75 & 0.0085 \\
\hline IL8 & Interleukin 8 & $\begin{array}{l}\text { Chemotaxis, Immune } \\
\text { response }\end{array}$ & 65.98 & 26.19 & 2.52 & 0.014 \\
\hline EGLN3 & $\begin{array}{l}\text { Egl-9 family hypoxia } \\
\text { inducible factor } 3\end{array}$ & $\begin{array}{l}\text { Apoptosis, regulation } \\
\text { of cell proliferation }\end{array}$ & 31.72 & 12.59 & 2.52 & 0.0085 \\
\hline MGST1 & $\begin{array}{l}\text { Microsomal glutathione } \\
\text { S-transferase } 1\end{array}$ & Redox homeostasis & 57.59 & 23.96 & 2.40 & 0.024 \\
\hline
\end{tabular}


Table 6: Genes with significantly lower expression in ARMD than MoP tissue. Gene expression levels in adverse reaction to metal debris (ARMD) and metal-on-plastic (MoP) samples are listed as reads per kilobase million (RPKM) values, and the differences as fold changes (FCs). $p$-values are adjusted by false discovery rate (FDR).

\begin{tabular}{|c|c|c|c|c|c|c|}
\hline Gene & Name & Function & \begin{tabular}{|l|l} 
RPKM \\
(ARMD)
\end{tabular} & $\begin{array}{l}\text { RPKM } \\
\text { (MoP) }\end{array}$ & FC & $\operatorname{adj} . p$ \\
\hline ACTA1 & actin, alpha 1 , skeletal muscle & Cell structure & 5.02 & 215.18 & -42.90 & 0.0085 \\
\hline DES & desmin & $\begin{array}{l}\text { Cell structure, } \\
\text { myofibroblast } \\
\text { development }\end{array}$ & 5.14 & 117.74 & -22.90 & 0.0085 \\
\hline HLA-B & $\begin{array}{l}\text { major histocompatibility complex, } \\
\text { class I, B }\end{array}$ & Immune response & 4.44 & 38.73 & -8.72 & 0.036 \\
\hline HLA-DRA & $\begin{array}{l}\text { major histocompatibility complex, } \\
\text { class II, DR alpha }\end{array}$ & Immune response & 11.15 & 78.83 & -6.85 & 0.024 \\
\hline SERPINA3 & serpin family A member 3 & Inflammatory response & 13.54 & 70.71 & -5.22 & 0.0085 \\
\hline CCL21 & C-C motif chemokine ligand 21 & \begin{tabular}{|l|} 
T cell chemotaxis, \\
osteoclast development
\end{tabular} & 17.69 & 87.55 & -4.95 & 0.024 \\
\hline RPS28 & ribosomal protein $\$ 28$ & Ribosome component & 110.94 & 406.39 & -3.66 & 0.0085 \\
\hline CHI3L1 & chitinase 3 like 1 (=YKL-40) & Inflammatory response & 118.76 & 407.95 & -3.44 & 0.0085 \\
\hline TPSB2 & tryptase beta 2 & Proteolysis & 17.79 & 57.86 & -3.25 & 0.047 \\
\hline PTGES & prostaglandin E synthase & Inflammatory response & 14.36 & 43.64 & -3.04 & 0.014 \\
\hline GSTT1 & glutathione S-transferase theta 1 & Redox homeostasis & 27.14 & 81.94 & -3.02 & 0.023 \\
\hline MT1E & metallothionein & Metal ion binding & 109.39 & 319.36 & -2.92 & 0.0085 \\
\hline RBP4 & retinol binding protein 4 & Glucose metabolism & 93.62 & 262.29 & -2.80 & 0.0085 \\
\hline TREM2 & $\begin{array}{l}\text { triggering receptor expressed on } \\
\text { myeloid cells } 2\end{array}$ & $\begin{array}{l}\text { Immune response, } \\
\text { osteoclast development }\end{array}$ & 133.31 & 347.37 & -2.61 & 0.023 \\
\hline S100A8 & S100 calcium binding protein $\mathrm{A} 8$ & Inflammatory response & 145.95 & 377.50 & -2.59 & 0.024 \\
\hline AEBP1 & AE binding protein 1 & $\begin{array}{l}\text { Skeletal system } \\
\text { development, } \\
\text { myofibroblast } \\
\text { development }\end{array}$ & 556.55 & 1431.68 & -2.57 & 0.024 \\
\hline CKB & creatine kinase $\mathrm{B}$ & \begin{tabular}{|l|} 
Energy homeostasis, \\
osteoclast development
\end{tabular} & 25.85 & 65.07 & -2.52 & 0.24 \\
\hline TNC & tenascin C & ECM organization & 41.74 & 97.90 & -2.35 & 0.039 \\
\hline LPL & lipoprotein lipase & Lipid metabolism & 11.17 & 27.44 & -2.34 & 0.0141 \\
\hline GGT5 & gamma-glutamyltransferase 5 & $\begin{array}{l}\text { Redox homeostasis, } \\
\text { inflammatory response }\end{array}$ & 23.00 & 53.56 & -2.33 & 0.019 \\
\hline COX6B1 & cytochrome c oxidase subunit 6B1 & Redox metabolism & 80.79 & 185.80 & -2.30 & 0.032 \\
\hline
\end{tabular}




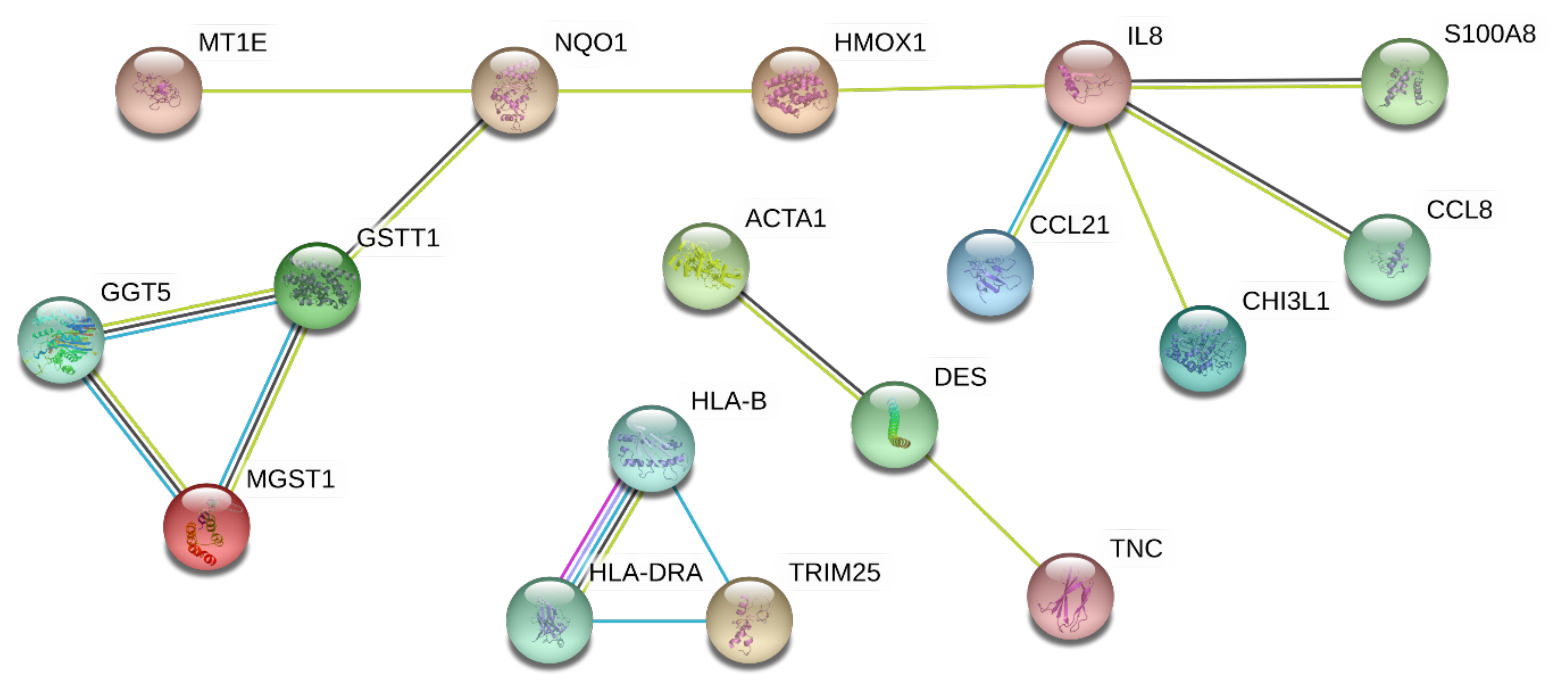

Figure 2: Interactions among the genes that were differentially expressed in ARMD vs MoP tissue. Significantly up- and downregulated genes in ARMD vs OA tissue were studied with STRING. Genes with no interactions are excluded from the graph. Colors of the edges: green = activation, blue = binding, black $=$ chemical reaction, red $=$ inhibition, violet $=$ catalysis, pink $=$ posttranslational modification, yellow $=$ transcriptional regulation, grey $=$ other interaction . 


\section{Discussion}

When the pseudosynovial ARMD tissue from failed MoM implants was compared to synovial tissue from OA joints, a major difference in the gene expression profile was found while the differences between the ARMD reaction and the inflammatory reaction around failed MoP joints were less pronounced. Although these control tissues, i.e. OA synovium and MoP tissue, are not ideal in all aspects (though probably the best available), this study is the first attempt to understand the pathogenesis of the ARMD reaction by applying genome-wide expression analysis.

The list of genes with markedly different expression levels in ARMD and OA samples was found to contain a large number of genes involved in inflammatory response, cell proliferation, cellular metabolism and apoptosis. The inflammatory genes appear to be dominated by those involved in macrophage and lymphocyte-mediated responses, including several cytokines and chemokines, fitting to the current conception of the ARMD response [6,13,32]. Accordingly, functional categories (GO terms) included several leukocyte signaling pathways such as phospholipase C, PI3K and tyrosine kinase signaling.

Among the genes with largest differences in expression between ARMD and OA tissue, an interaction network centered on clusters of differentiation (CDs) CD2, CD52, CD53 and PRKACB (all strongly upregulated in ARMD samples) was discovered in the STRING analysis. Of these, CD2, CD52 and CD53 transduce signals from $T$ cell receptors $[33,34]$, and might thus mediate lymphocyte-mediated hypersensitivity reactions to metals. The immunoglobulin receptor genes FCGR2A, FCGR2B and FCER1G (also identified in the STRING analysis) may also participate in these reactions. However, as far as we know, no previous information about the role of any of these genes in ARMD reaction has been published. PRKACB is a serine/threonine protein kinase mediating CAMP signaling, with subsequent effects on a wide range of cellular processes, including proliferation, differentiation and inflammation $[35,36]$. Another, smaller network is centered on the pentose phosphate shunt -related gene phosphogluconate dehydrogenase (PGD), and includes genes related to carbohydrate, lipid and alcohol metabolism. Yet another network was found to be focused on aggrecan (ACAN), and included proteoglycan 4 (PRG4), growth and differentiation factor 5 (GDF5) and hyaluronan and 
proteoglycan link protein 1 (HAPLN1). These genes, traditionally most strongly associated with cartilage metabolism, also seem to be expressed at lower levels in other tissues [37]. All of these genes were higher in OA synovium than in ARMD tissue, possibly reflecting compensatory increased synthesis of extracellular matrix (ECM) components in fibrotic synovial tissue typical for advanced OA [38].

The pathogenesis of the ARMD reaction is thought to be driven by metal ions and particles derived from MoM implants $[8,39]$. These implants are made of cobalt-chromium alloys, with other metals such as molybdenum and tungsten present in smaller amounts [40]. Due to their very high specific strength and corrosion resistance, these alloys were initially thought to be ideal for biomedical applications [41]. However, especially when subjected to large mechanical stress and "edge loading" (head-cup contact patch extending over the cup rim), significant amounts of metal particles can be abraded from MoM implants into the surrounding tissues $[8,42]$. Cobalt nanoparticles and $\operatorname{Co}(\mathrm{II})$ and $\mathrm{Cr}(\mathrm{VI})$ ions appear to be especially toxic, with chromium particles and $\mathrm{Cr}(\mathrm{III})$ ions only becoming harmful at markedly greater concentrations $[43,44]$. In the literature, the biochemical mechanisms of cobalt-induced toxicity appear to be more comprehensively characterized than those of chromium. In addition, cobalt may activate macrophages directly through TLR4 [17]. It also modifies macrophage phenotype [45] and causes strong oxidative stress [46].

Cobalt is known to mimic hypoxic conditions in cells, inhibiting the degradation of the transcription factor hypoxia-inducible factor 1 alpha (HIF1A) in the proteasome [47] and, accordingly, high expression of hypoxiarelated genes was observed in ARMD samples. These genes include heme oxygenase 1 (HMOX1), NADPH quinone dehydrogenase 1 (NQO1), Egl-9 family hypoxia inducible factor 3 (EGLN3) and superoxide dismutase 2 (SOD2). HIF1A mRNA levels were not significantly different in either comparison. This was however expected, as hypoxia (as well as cobalt) is known to enhance HIF1A expression by inhibiting the degradation of the protein, not by affecting on transcription. Accordingly, enhanced HIF1A protein levels have been detected in ARMD tissue [18] and in macrophages exposured to cobalt [45]. In conclusion, the present results support the hypoxia mimicry hypothesis as a contributing factor in AMRD, but further studies are needed to understand the pathogenetic mechanisms in detail. 
When comparing gene expression in ARMD tissue to that in the inflammatory tissue around failed MoP joints, a relatively small number of significantly up- or downregulated genes were identified. This suggests that the pathophysiologies of these two reactions hold many similarities. Indeed, both reactions are thought to be particle driven, the other by polyethylene debris and the other by metal debris. Alarmins, endogenous factors that promote noninfective inflammation, may serve as an example of mechanisms involved in both responses. As compared to OA samples, ARMD tissue expressed increased levels of S100 calcium-binding protein A9 (S100A9), an alarmin that heterodimerizes with S100A8 to form calprotectin [48]. MoP tissue expressed S100A9 at equally high levels and in addition, the expression of S100A8 was higher in MoP than in ARDM tissue. Alarmin S100A8/S100A9 has been associated with a wide variety of inflammatory conditions, from arthritis [49] to lung injury [50]. In previous studies, alarmins in general have been linked to aseptic implant loosening [51,52]. Further studies are needed to understand their detailed role in the pathogenesis of the response.

There were also significant differences in the gene expression profiles between ARMD and MoP tissues that may provide insights into the mechanisms of the two reaction types. Among the genes upregulated in ARMD tissue compared to MoP, there were, perhaps expectedly, several associated with metal ion binding and redox homeostasis. These include ring finger protein 170 (RNF170), solute carrier family 40 member 1 (SLC40A1) and heme oxygenase 1 (HMOX1). The last of these is especially interesting, as it is a cytoprotective factor induced during hypoxia [53]. HMOX1 mediates heme catabolism [54], but also regulates the inflammatory response by inhibiting the activation and nuclear translocation of the inflammatory transcription factor NF-KB and by enhancing the production of anti-inflammatory cytokines [55]. Along with HMOX1, NAD(P)H quinone dehydrogenase (NQO1) and microsomal glutathione S-transferase 1 (MGST1) [56] (which were both enhanced in ARMD tissue) are likely compensatory mechanisms to combat the oxidative stress induced by metal ions.

List of the genes, which were higher in MoP than ARMD tissue contained also a number of inflammatory genes. These genes appear to be widely expressed by both macrophages and lymphocytes, and thus provide 
no specific information about the involvement of these cell types in the reaction. Interestingly, many genes stimulating the differentiation and functions of osteoclasts were more highly expressed in MoP tissue compared to ARMD. These include C-C motif chemokine ligand 21 (CCL21) [57], triggering receptor expressed on myeloid cells 2 (TREM2) [58] and creatine kinase B (CKB) [59]. Osteoclast-mediated bone resorption is a known feature of adverse reactions seen in MoM [60] and especially in MoP [22] joints, and polyethylene particles have been demonstrated to promote osteoclastic differentiation of mononuclear cells [61]. However, the precise roles of the aforementioned factors in these processes remain largely unknown. As far as we know, no comprehensive comparative analysis of gene expression in ARMD and MoP reactions has previously been published.

A possible weakness of the present study is that both ARMD and MoP samples were from around failed prostheses. This leaves open the possibility that part of the observed changes may be attributed to the normal tissue reaction following arthroplasty or to the particle driven reaction leading to implant failure in general, rather than specific metal (or plastic) debris evoked toxicity. An ideal control for ARMD tissue would have been pseudosynovial tissue from around a normally functioning MoM joint with no signs of ARMD. Obtaining such tissue from well-functioning joints is, however, not possible because there is no indication for revision surgery. In general, the present results should be interpreted considering these limitations.

The present results show that there is a widespread difference in gene expression between pseudosynovial ARMD tissue and synovial tissue from OA joint. In contrast, differences in gene expression between ARMD and MoP tissues were less pronounced and, interestingly, osteolytic genes were among those significantly upregulated in MoP tissue. The big picture, based on genome-wide expression analysis, shows that ARMD reaction has unique features including up-regulation of genes associated with redox homeostasis, metal ion binding and transport, lymphocyte and macrophage activation, cellular metabolism and apoptosis. 


\section{Acknowledgements}

We wish to thank Mss. Meiju Kukkonen and Petra Miikkulainen for excellent technical assistance, as well as Mrs. Heli Määttä for skillful secretarial help.

\section{Funding sources}

The study was supported by the competitive research funding of Tampere University Hospital, Tampere, Finland.

\section{References}

[1] A. Eskelinen, V. Remes, I. Helenius, P. Pulkkinen, J. Nevalainen, P. Paavolainen, Total hip arthroplasty for primary osteoarthrosis in younger patients in the Finnish arthroplasty register. 4,661 primary replacements followed for 0-22 years, Acta Orthop. 76 (2005) 28-41. doi:10.1080/00016470510030292.

[2] M. Bhandari, M. Swiontkowski, Management of Acute Hip Fracture., N. Engl. J. Med. 377 (2017) 2053-2062. doi:10.1056/NEJMcp1611090.

[3] J.A. López-López, R.L. Humphriss, A.D. Beswick, H.H.Z. Thom, L.P. Hunt, A. Burston, C.G. Fawsitt, W. Hollingworth, J.P.T. Higgins, N.J. Welton, A.W. Blom, E.M.R. Marques, Choice of implant combinations in total hip replacement: systematic review and network meta-analysis, BMJ. (2017) j4651. doi:10.1136/bmj.j4651.

[4] A. Reito, L. Lehtovirta, O. Lainiala, K. Mäkelä, A. Eskelinen, Lack of evidence-the anti-stepwise introduction of metal-on-metal hip replacements., Acta Orthop. 88 (2017) 478-483. doi:10.1080/17453674.2017.1353794.

[5] S.-A.L. Ras Sørensen, H.L. Jørgensen, S.L. Sporing, J.B. Lauritzen, Revision rates for metal-on-metal hip resurfacing and metal-on-metal total hip arthroplasty - a systematic review, Hip Int. 26 (2016) 515-521. doi:10.5301/hipint.5000444.

[6] H.-G. Willert, G.H. Buchhorn, A. Fayyazi, R. Flury, M. Windler, G. Köster, C.H. Lohmann, Metal-onmetal bearings and hypersensitivity in patients with artificial hip joints. A clinical and histomorphological study, J. Bone Joint Surg. Am. 87 (2005) 28-36. doi:10.2106/JBJS.A.02039pp.

[7] M.H.L. Liow, Y.-M. Kwon, Metal-on-metal total hip arthroplasty: risk factors for pseudotumours and 
clinical systematic evaluation., Int. Orthop. 41 (2017) 885-892. doi:10.1007/s00264-016-3305-1.

[8] H. Dahlstrand, A. Stark, M.C. Wick, L. Anissian, N.P. Hailer, R.J. Weiss, Comparison of metal ion concentrations and implant survival after total hip arthroplasty with metal-on-metal versus metalon-polyethylene articulations, Acta Orthop. 88 (2017) 490-495. doi:10.1080/17453674.2017.1350370.

[9] A. Matthies, R. Underwood, P. Cann, K. Ilo, Z. Nawaz, J. Skinner, A.J. Hart, Retrieval analysis of 240 metal-on-metal hip components, comparing modular total hip replacement with hip resurfacing, J. Bone Joint Surg. Br. 93 (2011) 307-314. doi:10.1302/0301-620X.93B3.25551.

[10] H.S. Gill, G. Grammatopoulos, S. Adshead, E. Tsialogiannis, E. Tsiridis, Molecular and immune toxicity of CoCr nanoparticles in MoM hip arthroplasty., Trends Mol. Med. 18 (2012) 145-55. doi:10.1016/j.molmed.2011.12.002.

[11] H. Pandit, M. Vlychou, D. Whitwell, D. Crook, R. Luqmani, S. Ostlere, D.W. Murray, N.A. Athanasou, Necrotic granulomatous pseudotumours in bilateral resurfacing hip arthoplasties: evidence for a type IV immune response, Virchows Arch. An Int. J. Pathol. 453 (2008) 529-534. doi:10.1007/s00428-008-0659-9.

[12] K. Man, L.-H. Jiang, R. Foster, X.B. Yang, Immunological Responses to Total Hip Arthroplasty, J. Funct. Biomater. 8 (2017). doi:10.3390/jfb8030033.

[13] P. Campbell, E. Ebramzadeh, S. Nelson, K. Takamura, K. De Smet, H.C. Amstutz, Histological features of pseudotumor-like tissues from metal-on-metal hips, Clin. Orthop. Relat. Res. 468 (2010) 23212327. doi:10.1007/s11999-010-1372-y.

[14] S.B. Goodman, Wear particles, periprosthetic osteolysis and the immune system, Biomaterials. 28 (2007) 5044-5048. doi:10.1016/j.biomaterials.2007.06.035.

[15] G. Liu, T. Guo, Y. Zhang, N. Liu, J.J. Chen, J.J. Chen, J. Zhang, J. Zhao, Apoptotic pathways of macrophages within osteolytic interface membrane in periprosthestic osteolysis after total hip replacement, APMIS Acta Pathol. Microbiol. Immunol. Scand. 125 (2017) 565-578. doi:10.1111/apm.12679.

[16] A. Jonitz-Heincke, J. Tillmann, A. Klinder, S. Krueger, J.P. Kretzer, P.J. Høl, A.C. Paulus, R. Bader, The Impact of Metal Ion Exposure on the Cellular Behavior of Human Osteoblasts and PBMCs: In Vitro Analyses of Osteolytic Processes, Mater. (Basel, Switzerland). 10 (2017). doi:10.3390/ma10070734.

[17] L. Samelko, S. Landgraeber, K. McAllister, J. Jacobs, N.J. Hallab, TLR4 (not TLR2) dominate cognate TLR activity associated with CoCrMo implant particles, J. Orthop. Res. Off. Publ. Orthop. Res. Soc. 35 (2017) 1007-1017. doi:10.1002/jor.23368.

[18] L. Samelko, M.S. Caicedo, S.-J. Lim, C. Della-Valle, J. Jacobs, N.J. Hallab, Cobalt-alloy implant debris induce HIF-1 $\alpha$ hypoxia associated responses: a mechanism for metal-specific orthopedic implant failure, PLoS One. 8 (2013) e67127. doi:10.1371/journal.pone.0067127.

[19] T. Fujishiro, D.J.F. Moojen, N. Kobayashi, W.J.A. Dhert, T.W. Bauer, Perivascular and diffuse lymphocytic inflammation are not specific for failed metal-on-metal hip implants, Clin. Orthop. Relat. Res. 469 (2011) 1127-1133. doi:10.1007/s11999-010-1649-1. 
[20] T.D. Zaveri, N. V Dolgova, J.S. Lewis, K. Hamaker, M.J. Clare-Salzler, B.G. Keselowsky, Macrophage integrins modulate response to ultra-high molecular weight polyethylene particles and direct particle-induced osteolysis, Biomaterials. 115 (2017) 128-140. doi:10.1016/j.biomaterials.2016.10.038.

[21] O.N. Schipper, S.L. Haddad, P. Pytel, Y. Zhou, Histological Analysis of Early Osteolysis in Total Ankle Arthroplasty, Foot Ankle Int. 38 (2017) 351-359. doi:10.1177/1071100716682333.

[22] M. Sartori, F. Vincenzi, A. Ravani, S. Cepollaro, L. Martini, K. Varani, M. Fini, M. Tschon, RAW 264.7 co-cultured with ultra-high molecular weight polyethylene particles spontaneously differentiate into osteoclasts: an in vitro model of periprosthetic osteolysis, J. Biomed. Mater. Res. A. 105 (2017) 510520. doi:10.1002/jbm.a.35912.

[23] M. Wolfien, C. Rimmbach, U. Schmitz, J.J. Jung, S. Krebs, G. Steinhoff, R. David, O. Wolkenhauer, TRAPLINE: a standardized and automated pipeline for RNA sequencing data analysis, evaluation and annotation, BMC Bioinformatics. 17 (2016) 21. doi:10.1186/s12859-015-0873-9.

[24] E. Afgan, D. Baker, M. van den Beek, D. Blankenberg, D. Bouvier, M. Čech, J. Chilton, D. Clements, N. Coraor, C. Eberhard, B. Grüning, A. Guerler, J. Hillman-Jackson, G. Von Kuster, E. Rasche, N. Soranzo, N. Turaga, J. Taylor, A. Nekrutenko, J. Goecks, The Galaxy platform for accessible, reproducible and collaborative biomedical analyses: 2016 update, Nucleic Acids Res. 44 (2016) W3-W10. doi:10.1093/nar/gkw343.

[25] D. Blankenberg, A. Gordon, G. Von Kuster, N. Coraor, J. Taylor, A. Nekrutenko, Manipulation of FASTQ data with Galaxy, Bioinformatics. 26 (2010) 1783-1785. doi:10.1093/bioinformatics/btq281.

[26] D. Kim, G. Pertea, C. Trapnell, H. Pimentel, R. Kelley, S.L. Salzberg, TopHat2: accurate alignment of transcriptomes in the presence of insertions, deletions and gene fusions, Genome Biol. 14 (2013) R36. doi:10.1186/gb-2013-14-4-r36.

[27] C. Trapnell, D.G. Hendrickson, M. Sauvageau, L. Goff, J.L. Rinn, L. Pachter, Differential analysis of gene regulation at transcript resolution with RNA-seq, Nat Biotechnol. 31 (2012). doi:10.1038/nbt.2450.

[28] M.A. Gene Ontology Consortium, J. Clark, A. Ireland, J. Lomax, M. Ashburner, R. Foulger, K. Eilbeck, S. Lewis, B. Marshall, C. Mungall, J. Richter, G.M. Rubin, J.A. Blake, C. Bult, M. Dolan, H. Drabkin, J.T. Eppig, D.P. Hill, L. Ni, M. Ringwald, R. Balakrishnan, J.M. Cherry, K.R. Christie, M.C. Costanzo, S.S. Dwight, S. Engel, D.G. Fisk, J.E. Hirschman, E.L. Hong, R.S. Nash, A. Sethuraman, C.L. Theesfeld, D. Botstein, K. Dolinski, B. Feierbach, T. Berardini, S. Mundodi, S.Y. Rhee, R. Apweiler, D. Barrell, E. Camon, E. Dimmer, V. Lee, R. Chisholm, P. Gaudet, W. Kibbe, R. Kishore, E.M. Schwarz, P. Sternberg, M. Gwinn, L. Hannick, J. Wortman, M. Berriman, V. Wood, N. de la Cruz, P. Tonellato, P. Jaiswal, T. Seigfried, R. White, Gene Ontology Consortium, The Gene Ontology (GO) database and informatics resource, Nucleic Acids Res. 32 (2004) 258D-261. doi:10.1093/nar/gkh036.

[29] D.W. Huang, B.T. Sherman, R.A. Lempicki, Systematic and integrative analysis of large gene lists using DAVID bioinformatics resources, Nat. Protoc. 4 (2009) 44-57. doi:10.1038/nprot.2008.211.

[30] F. Supek, M. Bošnjak, N. Škunca, T. Šmuc, REVIGO Summarizes and Visualizes Long Lists of Gene Ontology Terms, PLoS One. 6 (2011) e21800. doi:10.1371/journal.pone.0021800. 
[31] D. Szklarczyk, A. Franceschini, S. Wyder, K. Forslund, D. Heller, J. Huerta-Cepas, M. Simonovic, A. Roth, A. Santos, K.P. Tsafou, M. Kuhn, P. Bork, L.J. Jensen, C. von Mering, STRING v10: proteinprotein interaction networks, integrated over the tree of life, Nucleic Acids Res. 43 (2015) D452. doi:10.1093/nar/gku1003.

[32] E.-L. Paukkeri, R. Korhonen, M. Hämäläinen, M. Pesu, A. Eskelinen, T. Moilanen, E. Moilanen, The Inflammatory Phenotype in Failed Metal-On-Metal Hip Arthroplasty Correlates with Blood Metal Concentrations., PLoS One. 11 (2016) e0155121. doi:10.1371/journal.pone.0155121.

[33] J. Leitner, D. Herndler-Brandstetter, G.J. Zlabinger, B. Grubeck-Loebenstein, P. Steinberger, CD58/CD2 Is the Primary Costimulatory Pathway in Human CD28-CD8+ T Cells, J. Immunol. (Baltimore, Md. 1950). 195 (2015) 477-487. doi:10.4049/jimmunol.1401917.

[34] S.R. Mohan, M.J. Clemente, M. Afable, H.N. Cazzolli, N. Bejanyan, M.W. Wlodarski, A.E. Lichtin, J.P. Maciejewski, Therapeutic implications of variable expression of CD52 on clonal cytotoxic T cells in CD8+ large granular lymphocyte leukemia, Haematologica. 94 (2009) 1407-1414. doi:10.3324/haematol.2009.009191.

[35] L.V. Moen, Z. Sener, R. Volchenkov, A.C. Svarstad, A.M. Eriksen, H.L. Holen, B.S. Skålhegg, Ablation of the $C \beta 2$ subunit of PKA in immune cells leads to increased susceptibility to systemic inflammation in mice, Eur. J. Immunol. 47 (2017) 1880-1889. doi:10.1002/eji.201646809.

[36] J.M. Steichen, G.H. Iyer, S. Li, S.A. Saldanha, M.S. Deal, V.L. Woods, S.S. Taylor, Global consequences of activation loop phosphorylation on protein kinase A, J. Biol. Chem. 285 (2010) 3825-3832. doi:10.1074/jbc.M109.061820.

[37] L. Fagerberg, B.M. Hallström, P. Oksvold, C. Kampf, D. Djureinovic, J. Odeberg, M. Habuka, S. Tahmasebpoor, A. Danielsson, K. Edlund, A. Asplund, E. Sjöstedt, E. Lundberg, C.A.-K. Szigyarto, M. Skogs, J.O. Takanen, H. Berling, H. Tegel, J. Mulder, P. Nilsson, J.M. Schwenk, C. Lindskog, F. Danielsson, A. Mardinoglu, Å. Sivertsson, K. von Feilitzen, M. Forsberg, M. Zwahlen, I. Olsson, S. Navani, M. Huss, J. Nielsen, F. Ponten, M. Uhlén, Analysis of the Human Tissue-specific Expression by Genome-wide Integration of Transcriptomics and Antibody-based Proteomics, Mol. Cell. Proteomics. 13 (2014) 397-406. doi:10.1074/mcp.M113.035600.

[38] T. Hardingham, Extracellular matrix and pathogenic mechanisms in osteoarthritis, Curr. Rheumatol. Rep. 10 (2008) 30-36. doi:10.1007/s11926-008-0006-9.

[39] P.A. Revell, Biological causes of prosthetic joint failure*Note: This chapter is an updated version of Chapter 15 from the first edition of Joint replacement technology edited by P. A. Revell and published by Woodhead Publishing 2008*, in: Jt. Replace. Technol., Elsevier, 2014: pp. 298-369. doi:10.1533/9780857098474.3.298.

[40] T.M. Devine, F.J. Kummer, J. Wulff, Wrought cobalt-chromium surgical implant alloys, J. Mater. Sci. 7 (1972) 126-128. doi:10.1007/BF00549560.

[41] D.J.S. Hyslop, A.M. Abdelkader, A. Cox, D.J. Fray, Electrochemical synthesis of a biomedically important Co-Cr alloy, Acta Mater. 58 (2010) 3124-3130. doi:10.1016/j.actamat.2010.01.053.

[42] R.J. Underwood, A. Zografos, R.S. Sayles, A. Hart, P. Cann, Edge loading in metal-on-metal hips: low 
clearance is a new risk factor., Proc. Inst. Mech. Eng. H. 226 (2012) 217-26.

doi:10.1177/0954411911431397.

[43] M. Akbar, J.M. Brewer, M.H. Grant, Effect of chromium and cobalt ions on primary human lymphocytes in vitro, J. Immunotoxicol. 8 (2011) 140-149. doi:10.3109/1547691X.2011.553845.

[44] Y.-M. Kwon, Z. Xia, S. Glyn-Jones, D. Beard, H.S. Gill, D.W. Murray, Dose-dependent cytotoxicity of clinically relevant cobalt nanoparticles and ions on macrophages in vitro., Biomed. Mater. 4 (2009) 25018. doi:10.1088/1748-6041/4/2/025018.

[45] M. Kumanto, E.-L. Paukkeri, R. Nieminen, E. Moilanen, Cobalt(II) Chloride Modifies the Phenotype of Macrophage Activation, Basic Clin. Pharmacol. Toxicol. 121 (2017) 98-105. doi:10.1111/bcpt.12773.

[46] K. Jomova, M. Valko, Advances in metal-induced oxidative stress and human disease, Toxicology. 283 (2011) 65-87. doi:10.1016/j.tox.2011.03.001.

[47] A. Nyga, A. Hart, T.D. Tetley, Importance of the HIF pathway in cobalt nanoparticle-induced cytotoxicity and inflammation in human macrophages, Nanotoxicology. 9 (2015) 905-917. doi:10.3109/17435390.2014.991430.

[48] F.S. Lehmann, E. Burri, C. Beglinger, The role and utility of faecal markers in inflammatory bowel disease, Therap. Adv. Gastroenterol. 8 (2015) 23-36. doi:10.1177/1756283X14553384.

[49] J. Austermann, S. Zenker, J. Roth, S100-alarmins: potential therapeutic targets for arthritis, Expert Opin. Ther. Targets. 21 (2017) 738-750. doi:10.1080/14728222.2017.1330411.

[50] D. Chakraborty, S. Zenker, J. Rossaint, A. Hölscher, M. Pohlen, A. Zarbock, J. Roth, T. Vogl, Alarmin S100A8 Activates Alveolar Epithelial Cells in the Context of Acute Lung Injury in a TLR4-Dependent Manner., Front. Immunol. 8 (2017) 1493. doi:10.3389/fimmu.2017.01493.

[51] Y. Tamaki, Y. Takakubo, K. Goto, T. Hirayama, K. Sasaki, Y.T. Konttinen, S.B. Goodman, M. Takagi, Increased expression of toll-like receptors in aseptic loose periprosthetic tissues and septic synovial membranes around total hip implants., J. Rheumatol. 36 (2009) 598-608. doi:10.3899/jrheum.080390.

[52] L. Samelko, S. Landgraeber, K. McAllister, J. Jacobs, N.J. Hallab, Cobalt Alloy Implant Debris Induces Inflammation and Bone Loss Primarily through Danger Signaling, Not TLR4 Activation: Implications for DAMP-ening Implant Related Inflammation, PLoS One. 11 (2016) e0160141. doi:10.1371/journal.pone.0160141.

[53] J.A. Neubauer, J. Sunderram, Heme oxygenase-1 and chronic hypoxia, Respir. Physiol. Neurobiol. 184 (2012) 178-185. doi:10.1016/j.resp.2012.06.027.

[54] M. Exner, E. Minar, O. Wagner, M. Schillinger, The role of heme oxygenase-1 promoter polymorphisms in human disease, Free Radic. Biol. Med. 37 (2004) 1097-1104. doi:10.1016/J.FREERADBIOMED.2004.07.008.

[55] C.A. Piantadosi, C.M. Withers, R.R. Bartz, N.C. MacGarvey, P. Fu, T.E. Sweeney, K.E. Welty-Wolf, H.B. Suliman, Heme Oxygenase-1 Couples Activation of Mitochondrial Biogenesis to Anti-inflammatory Cytokine Expression, J. Biol. Chem. 286 (2011) 16374-16385. doi:10.1074/jbc.M110.207738. 
[56] J. Shi, H.L. Karlsson, K. Johansson, V. Gogvadze, L. Xiao, J. Li, T. Burks, A. Garcia-Bennett, A. Uheida, M. Muhammed, S. Mathur, R. Morgenstern, V.E. Kagan, B. Fadeel, Microsomal glutathione transferase 1 protects against toxicity induced by silica nanoparticles but not by zinc oxide nanoparticles, ACS Nano. 6 (2012) 1925-1938. doi:10.1021/nn2021056.

[57] J. Lee, C. Park, H.J. Kim, Y.D. Lee, Z.H. Lee, Y.W. Song, H.-H. Kim, Stimulation of osteoclast migration and bone resorption by C-C chemokine ligands 19 and 21, Exp. Mol. Med. 49 (2017) e358. doi:10.1038/emm.2017.100.

[58] M.B. Humphrey, M.R. Daws, S.C. Spusta, E.C. Niemi, J.A. Torchia, L.L. Lanier, W.E. Seaman, M.C. Nakamura, TREM2, a DAP12-associated receptor, regulates osteoclast differentiation and function, J. Bone Miner. Res. Off. J. Am. Soc. Bone Miner. Res. 21 (2006) 237-245. doi:10.1359/JBMR.051016.

[59] E.-J. Chang, J. Ha, F. Oerlemans, Y.J. Lee, S.W. Lee, J. Ryu, H.J. Kim, Y. Lee, H.-M. Kim, J.-Y. Choi, J.Y. Kim, C.S. Shin, Y.K. Pak, S. Tanaka, B. Wieringa, Z.H. Lee, H.-H. Kim, Brain-type creatine kinase has a crucial role in osteoclast-mediated bone resorption, Nat. Med. 14 (2008) 966-972. doi:10.1038/nm.1860.

[60] L. Dong, R. Wang, Y.-A. Zhu, C. Wang, H. Diao, C. Zhang, J. Zhao, J. Zhang, Antisense oligonucleotide targeting TNF-alpha can suppress Co-Cr-Mo particle-induced osteolysis, J. Orthop. Res. Off. Publ. Orthop. Res. Soc. 26 (2008) 1114-1120. doi:10.1002/jor.20607.

[61] F. Veronesi, M. Tschon, M. Fini, Gene Expression in Osteolysis: Review on the Identification of Altered Molecular Pathways in Preclinical and Clinical Studies, Int. J. Mol. Sci. 18 (2017) 499. doi:10.3390/ijms18030499. 